\title{
Dr. mont. Janet Zaph Briggs und ihre Rennofenversuche in Stanford, Ca. 1933
}

\section{G. Sperl}

Lehrstuhl für Materialphysik, Montanuniversität Leoben, Leoben, Österreich

Eingegangen 17. August 2018; angenommen 25. August 2018; online publiziert 13. November 2018

Zusammenfassung: Die experimentelle Archäometallurgie des Eisens, die naturwissenschaftliche Untersuchung des antiken Eisenwesens, war schon 1977 am Hüttenberger Erzberg durch Schmelzversuche begonnen worden. In USA war die junge Studentin Janet Zaph Briggs (1912-1974) mit ihrer Diplomarbeit an der Leland-Stanford University in Kalifornien die erste, die dies, noch wenig durch grundlegendenmetallurgische Theorien befasst, 1933 zur Aufgabe ihrer Diplomarbeit machte. Die Schmelzversuche brachten direkt nur wenig schmiedbares Eisen, aber die Erfahrungen und die Untersuchungen über die Besonderheit der Korrosion des historischen Eisens waren wertvoll und wurden mit dem Titel „Engineer" durch die Universität belohnt. Ihre Vorliebe für Österreich brachten sie auch dazu, in Wien mit einer Dissertation zu beginnen, die dann 1936 in Leoben mit dem „Dr. mont." belohnt wurde. Beruflich war sie später be der Climax Molybdenum Company mit Sitz in New York wissenschaftlich erfolgreich.

Schlüsselwörter: Janet Z. Briggs, Diplomarbeit, Archäometallurgie, Eisen

Janet Zaph Briggs (1912-1974) Stanford-Leoben, and the Metallurgical Archaeometry of Iron

Abstract: The metallurgical archaeometry of iron, the scientific research on the history of iron, started with smelting experiments and analyses of their slags. In Austria, the earliest experiments were documented in 1877; in America, first experiments are described in the thesis, published 1933 at the Leland-Stanford University in California, by a young student of mining and metallurgy, Janet Zaph Briggs (1912-1974).

Even if the smelting experiments were not very successful, the experiences gained from smelting iron and the tests

\footnotetext{
G. Sperl ( $\square)$

Lehrstuhl für Materialphysik,

Montanuniversität Leoben,

Jahnstrasse 12/l,

8700 Leoben, Österreich

gerhard.sperl@unileoben.ac.at
}

on corrosion provided a completely new view on early metallurgy of iron in the USA. After receiving her doctorate in Leoben in 1936, Janet Briggs became a very successful researcher at the Climax Molybdenum Company.

Keywords: Janet Zaph Briggs (1912-1974), Thesis, Archaeometallurgy, Iron

\section{Einleitung}

Die Idee, das historische Rennverfahren zur direkten Erzeugung von schmiedbarem Stahl aus dem Erz durch Schmelzversuche nachzuempfinden, ist im Alpenraum schon 1877 am Magdalensberg erstmals verwirklicht worden [1]. Anstoß war der Fund von Schmelzgruben, deren Brauchbarkeit zur Eisengewinnung man durch Schmelzversuche beweisen wollte. Tatsächlich wurde schmiedbares Eisen gewonnen und zu Geräten aus diesem Stahl ausgeschmiedet.

Erst in der Zwischenkriegszeit folgten weitere Schmelzversuche; in dieser Tradition ist auch die Diplomarbeit von Janet Zaph Briggs (1912-1974) in Stanford, Kalifornien 1933 [2] zu sehen, mit der sie den Titel eines Bergingenieurs (Mining Engineer) erwarb [NB1]. Wie in der in Leoben 1936 vorgelegten Dissertation [3] war ein Teil ihres Interesses der Frage der Korrosion gewidmet.

\section{Kurzbiographie}

Janet Zaph Briggs wurde 1912 in Santa Ana, Kalifornien, geboren [4]. Der Vater, George Briggs (1861-1946), heiratete 1892 Eva Potts in Arizona. Janet hatte zwei Geschwister, die Familie übersiedelte 1909 nach Santa Ana in Kalifornien. George Briggs war ein enthusiastischer Jäger, engagierte sich auch in Politik und im örtlichen Bankwesen.

Janet Z. Briggs erreichte den Grad eines Bachelors an der "Leland Stanford Junior University" in Palo Alto 1931 und schloss die Universität 1933 mit dem Grad eines „Engineer." Damals war es auch in Stanford nicht selbstverständlich, dass Studentinnen ein technisches Fach studierten, Briggs 
war hier die erste und wurde aber bald voll als Kollegin anerkannt. In ihrer Studienbeschreibung finden sich außer den üblichen Engineering-Kursen auch seit 1928 Deutsch, und dies blieb bis zum Studienende 1933 in der Liste. In ihrer Studienzeit war der Bruder des US-Präsidenten Herbert Clark Hoover, dem 1933 in Leoben die Ehrendoktorwürde verliehen wurde [5], Theodor J. Hoover, Dekan der Engineering-Fakultät, der sie auf ein Postgraduate Studium in Europa vorbereitete [NB2].

\section{Ihre wissenschaftlichen Arbeiten}

\subsection{Diplomarbeit Stanford 1833 [2]:}

"A short Study of the Making, Working, and Properties of Ancient Iron" (\%-Angaben immer in Massen- bzw. Gewichts-\%, Seitenzahlen beziehen sich auf die Diplomarbeit [2]).

\subsubsection{Briggs begründet die Aufgabe ihrer Arbeit in Kap. 1:}

The object of this experiment is threefold: first, to determine how practical the methods for iron manufacture given by the archaeologists are; second, to test the iron made by ancient methods for corrosion resistance; and third, to try to find the reason for the corrosion resistance of ancient iron.

This subject is of academic interest only. Many accounts have been given of the ancient iron furnaces and their operation, but there seems to be no report of a metallurgist producing iron in a direct reduction furnace of ancient design and then testing the iron for corrosion resistance.

Interessant ist hier, dass sie ausdrücklich nicht nur die Herstellung, sondern auch das bessere Korrosionsverhalten des Rennofeneisens untersucht und hier tatsächlich die erste ist, während bei den Schmelzversuchen Gundacker Graf Wurmbrand am Hüttenberger Erzberg schon 1877 erfolgreich war [1]. Ihre Versuche beginnen mit Tests an Hämatit und Magnetit im Lagerfeuer (Abb. 1), doch bald geht sie auf einen Schachtofen über, der in Anlehnung an die indische Ofenbeschreibung (S. 17) gebaut wird. (Abb. 2).

Den Versuchen (S. 79-84) geht eine umfangreiche Literaturrecherche voraus, deren Ergebnis auf 56 Seiten (S. 2-58) im Detail beschrieben wird und auf insgesamt 231 Literaturstellen basiert. Im Anhang (S. I-XVIII) finden sich auch die Beschreibungen von Untersuchungen an 141 Eisenobjekten aus der Literatur (auf 8 Doppelseiten).

Kennzeichnend für diese Sammlung sei die Beschreibung der Eisernen Säule in Delhi, Indien, 4. Jahrhundert n. Chr. (Abb. 3), die, wie die Literaturzitate in der rechten Spalte zeigen, bereits bis 1933 viermal in der Literatur behandelt wurde. Heute weiß man, dass diese Säule hohl ist, mit einer Wandstärke von etwa $5 \mathrm{~cm}$ aus etwa 200 Luppenstücken gefertigt wurde und nur 2 Tonnen wiegt [6]. Briggs findet auch schon damals, dass die Perlen aus Meteoreisen der Gerzeh-Kultur (Ägypten 3500-3200 v. Chr.) die ältesten vom Menschen bearbeiteten Eisenobjekte sind und dass

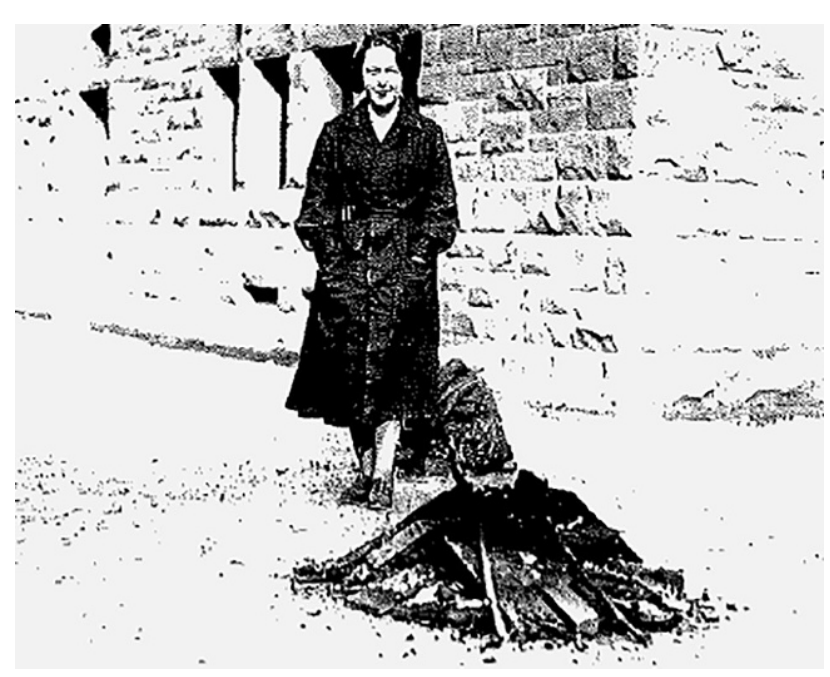

Abb. 1: J.Z. Briggs beim Versuch im Lagerfeuer (V2 in [2])

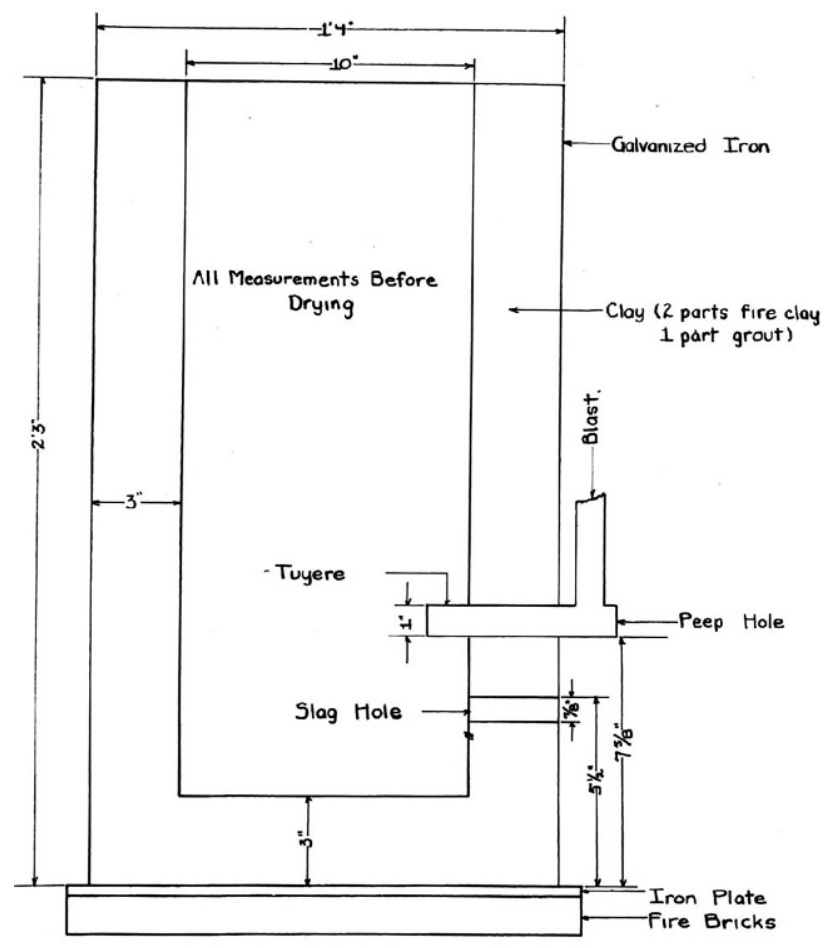

FURNACE

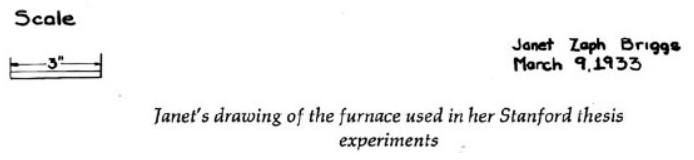

Abb. 2: Der Rennofen (Schachtofen) für die Versuche (\#3-7)

das 1937 in der Cheops-Pyramide (um 2600 erbaut) gefundene Eisenblech sicher nicht antik ist.

Die Versuche zur Reduktion des Eisens aus dem Erz werden an lokalen Erzen (Hämatit 69,36\% Fe, 0,29\% $\mathrm{SiO}_{2}$, Magnetit $60,94 \% \mathrm{Fe}, 8,93 \% \mathrm{SiO}_{2}$ ) durchgeführt. (S. 59/2). Als Versuch V1 wurden beide Erze gesetzt und $7 \mathrm{~h}$ in einer 
Abb. 3: Gupta-Säule in Delhi (Indien) bei Briggs 1933 [2], Appendix, S. XIV/XV, Nr. 110

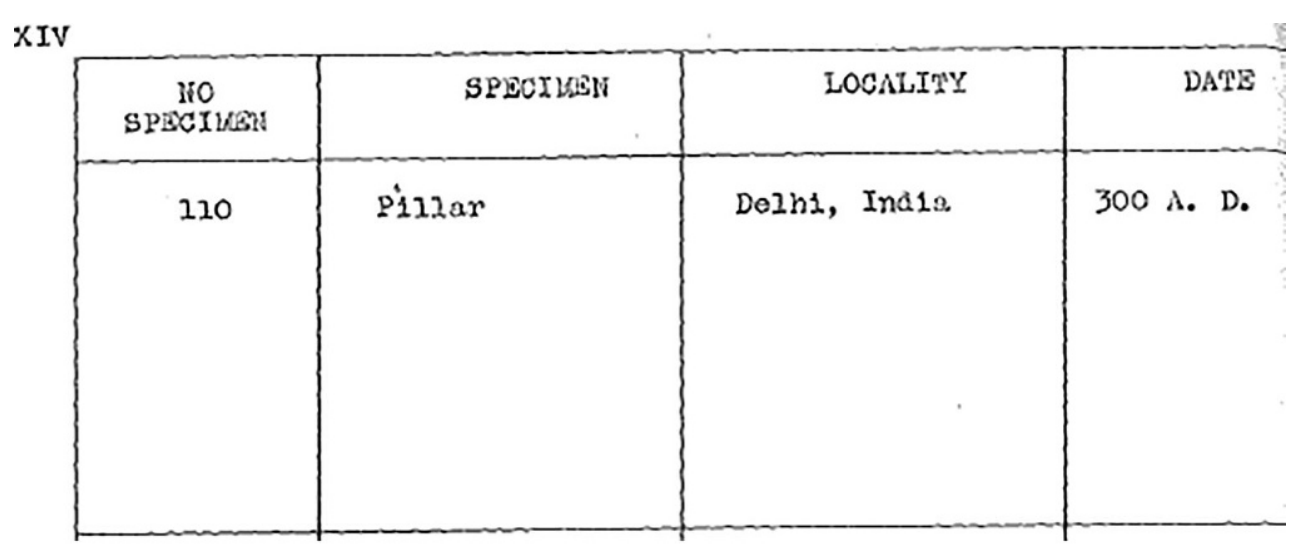

a

\begin{tabular}{|c|c|c|c|}
\hline PRBSERVATION & ANALYSIS & REISARKS & $\begin{array}{c}\text { NO } \\
\text { RERERENCES }\end{array}$ \\
\hline $\begin{array}{l}\text { Perfect above } \\
\text { Bround. Some } \\
\text { corrosion below }\end{array}$ & $\begin{array}{ll}c & .08 \\
\text { s } & .006 \\
\text { lsn } & n 11 \\
\text { Cu } & .034 \\
\text { s1 } & .046 \\
N & .032 \\
\text { p } & .124 \\
\text { fe } & 99.72\end{array}$ & $\begin{array}{l}24^{\prime} \text { tall. } 20^{\prime \prime} \text { under } \\
\text { ground. 12a" }= \\
\text { dian. at top. } \\
\text { l6s" a dam. at } \\
\text { botton. } \\
\text { innolnted with } \\
\text { butter onco a } \\
\text { year. } \\
\text { Inscriptions clear } \\
\text { Few inclusions. } \\
\text { Yoliow patina. }\end{array}$ & $56,62,78,83$ \\
\hline
\end{tabular}

Schmiedeesse erhitzt; die Erze hatten sich nicht verändert. Für Versuch V2 wurden wieder gemischte Erze gesetzt, es wurde ein größeres Feuer (mit Holz und Holzkohle (Abb. 1) und unter mäßigem Wind $3 \mathrm{~h}$ geglüht, ebenfalls ohne Änderung der Erze.

Für die Versuche 3 bis 7 (bei Briggs \#1-5) wurde ein Schachtofen nach den Vorstellungen zu dem indischen Schachtofen [7] leicht modifiziert (Abb. 2) gewählt: Der innere lichte Durchmesser war $254 \mathrm{~mm}\left(10^{\prime \prime}\right)$, die lichte Bauhöhe ca. $600 \mathrm{~mm}$ (2ft), der Innendurchmesser der Winddüse $25 \mathrm{~mm}\left(1^{\prime \prime}\right)$, die Wandstärke (aus Ton und Mörtel) $75 \mathrm{~mm}$ (3"). Für V3-5 wurde Hämatit, für V6-7 Magnetit eingesetzt.

Nach Auswertung der Angaben auf S. 67 wurde bei Versuch 3 (\#1) der sorgfältig getrocknete Ofen mit Holzkohle gefüllt (ca. $60 \mathrm{dm}^{3}>\sim 12 \mathrm{~kg}$ ) und diese niedergebrannt; es wurden dann 14,2 Pounds $(6,4 \mathrm{~kg})$ Erz innerhalb einer Stunde gesetzt, was einen Satz von etwa $2 \mathrm{~kg}$ Holzkohle je kg Erz bedeutet; unter Berücksichtigung der Anheizphase mit Holzkohle liegt der Wert $4 \mathrm{~kg}$ je $\mathrm{kg}$ Erz, für erfolgreiche Schmelzversuche durchaus sinnvolle Werte. Der Verbrauch an Holzkohle lag hier bei etwa $12 \mathrm{~kg}$ je Stunde (Gestellbelastung $60 \mathrm{~kg} \mathrm{HK} / \mathrm{m}^{2}$.h). Es wurde ein Schmelzblock von etwa $12 \mathrm{~cm}$ Außenmaß erzeugt, der allerdings unter dem Hammer in kleine Stücke zerbarst, in denen kleinere Eisenfladen enthalten waren.

Bei Versuch V4 (\#2) wurde Hämatit und Retourschlacke von Versuch V3 eingesetzt. Man heizte länger und hatte
Probleme mit Ofenansätzen; das Ergebnis war aber enttäuschend (0,79\% Fe-met!); nach Meinung von Briggs hatte Rückoxidation stattgefunden. Für Versuch V $5+6$ vergrößerte man die Winddüse. Bei V5 (\#3) wurde Hämatit, bei V6 (\#4) Magnetit eingesetzt. Der letzte Versuch (V7/\#5) wurde zwar heißer gefahren, ergab aber wegen Verschlackung auch ein unbefriedigendes Ergebnis.

Als man den Schlackenblock von Versuch (\#2) in der Schmiede erhitzte, verbrannte die eingeschlossene Holzkohle und die Schlacke wurde flüssig ausgetrieben. Man schmiedete diese kleinen Eisenluppen im Gesamtgewicht von 1 Pfund $(0,45 \mathrm{~kg})$ für weitere Untersuchungen zusammen zu einer Probe.

Ergebnis der Versuche: Die chemische Untersuchung des Eisens aus \#4 ergab: C 0,41\%, Mn 0,42\%, Si 0,06\%, S $0,005 \%, P$ P $100 \%$.

Der relativ hohe $\mathrm{Mn}-$ Gehalt zeigt, dass in der Probe noch Schlackeneinschlüsse waren (der Magnetit hatte 0,118\% $\mathrm{Mn})$; der niedrige $\mathrm{SiO}_{2}$-Gehalt zeigt das Manko dieser Versuche, das Fehlen von Zuschlägen, die Erze waren ohne schlackenbildende Zuschläge eingesetzt worden.

Zum Thema Korrosion: Es wurde in diesem Zusammenhang die Frage gestellt, warum manche antiken Eisenobjekte so gut erhalten sind. Dem wollte Briggs mit einer kleinen Versuchsreihe auf den Grund gehen. Die Korrosionstests wurden an zwei antiken Stücken und den Proben 
aus den Schmelzversuchen durchgeführt, wobei ein moderner Stahl als Vergleichswerkstoff diente. Praktisch immer war der Gewichtsverlust bei den antiken Stücken und dem Testproben deutlich niedriger als für den modernen Stahl. Briggs gibt als Hauptursache den Phosphorgehalt der antiken Stücke an (S. 87).

\subsubsection{Von J. Z. Briggs verwendete Literatur}

Der Anhang „Bibliography“ mit 14 Seiten umfasst 231 Literaturstellen in alphabetischer Reihenfolge der Autoren; die älteste zitierte Arbeit ist Percys Metallurgy, Iron and Steel 1864, aber im Text wird u. a. auch Homer, Aristoteles, Dioscurides und Plinius zitiert. Das häufigste Stichwort ist neben Iron und Steel "Phosphor" in 24 Titeln.

\subsection{Dissertation Leoben 1936 [3]}

„Versuche über das anodische Verhalten von Chrom und V2A-Stahl“.

Janet Briggs (Abb. 4) kam wohl nicht nur wegen ihrer Deutschkenntnisse, sondern auch durch ihre Beziehungen zu den Hoovers nach Österreich und zur Leobner Montanuniversität [NB2]. Bereits einen Monat nach ihrer Graduierung zum „Engineer" an der Stanford-Universität kam sie im Juli 1933 nach Österreich. Für diesen Aufenthalt hat Robert $\mathrm{Q}$. Barr in seinem Buch [4] viele Details angegeben. Neben ihrer wissenschaftlichen Arbeit an der Technischen Universität Wien bereiste sie fast ganz Europa, und auch eine Reise nach Ägypten war dabei.

Wie L. Jontes [8] schreibt, studierte Briggs an der Wiener TU von 1933 bis 1935 bei Prof. Wolf Müller und legte dann in Leoben am 11. Dezember 1935 an der Montanistischen Hochschule Leoben ihr Rigorosum ab und wurde Anfang 1936 unter dem Grazer Rektor Prof. Hans Paul und dem Leobener Dekan Prof. Wilhelm Petraschek sen. zum Dr. mont. promoviert (Abb. 5). Die Leobner Schule war 1935 mit der Technischen Hochschule Graz zusammengelegt worden. Einen ersten Studienabschnitt erledigte sie wahrscheinlich in Wien, während das Fachstudium in Leoben erfolgte. Ihre Dissertation steht in der Bibliothek der Montanuniversität und zeigt einen Aufbau, der schon bei der Diplomarbeit angewendet worden war: Es beginnt mit sehr einfachen Versuchen zur Oxidation von Chrom, um dann immer detaillierter in das Hauptthema des V2A-Stahles, hier meist Chrom-Nickel-Stahl 18/8 bezeichnet, einzugehen. Die Dissertation (in zwei Bänden) ist sehr detailreich und durch zahlreiche chemische Bewertungen unterlegt. In [4] wird auch eingehend auf das Privatleben, vor allem in Österreich, Bezug genommen, wofür die ausführlichen Tagebücher herangezogen werden konnten. Sie beschreibt auch humorvoll die Leobener Promotionszeremonie am 1. Februar 1936.

\section{Beruflicher Werdegang}

Ing. Dr. mont. Janet Zaph Briggs verließ am 22. August 1936 Europa in Hamburg an Bord der „Vancouver" und kam da-

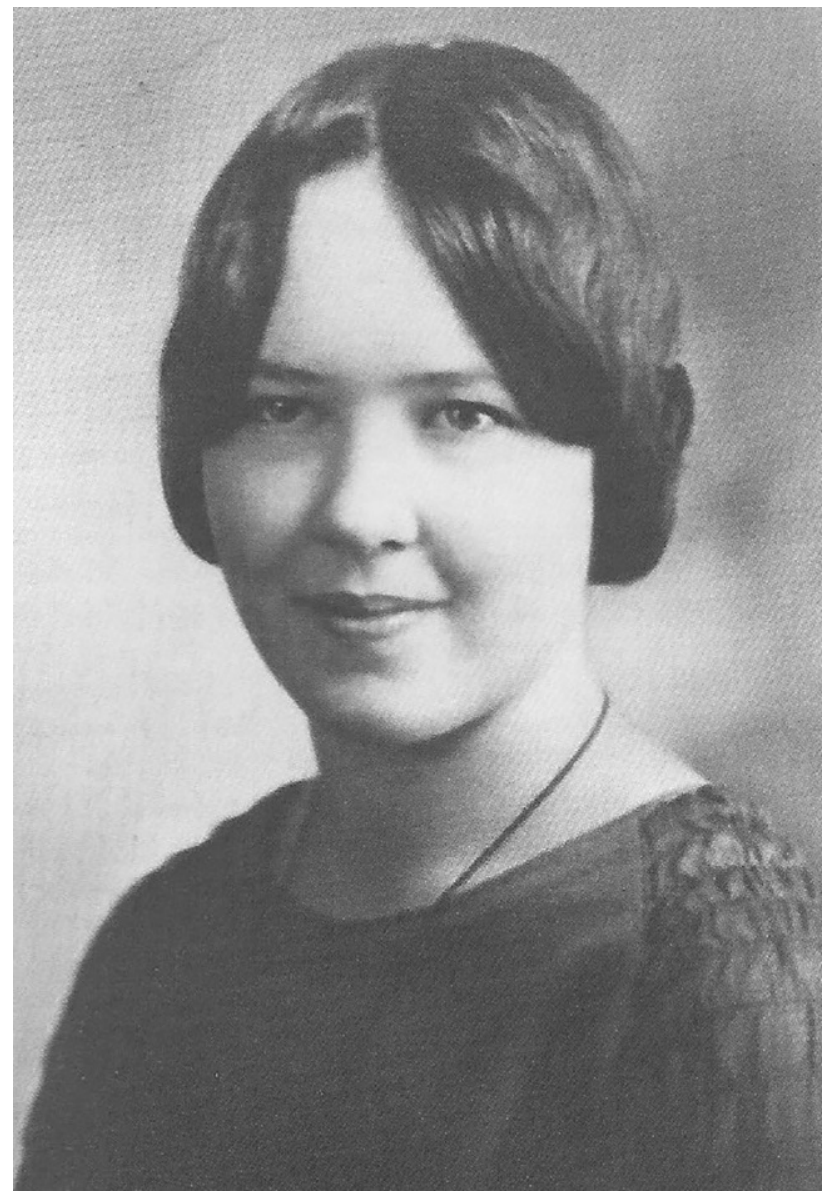

Abb. 4: Janet Zaph Briggs in ihrer Zeit in Österreich (1933-1936)

mit am 25. September 1936 in Los Angeles an. Sie versuchte nun durch zahlreiche Bewerbungen eine passende Stellung zu finden, was für weibliche Bewerber auf dem technischen Gebiet Schwierigkeiten zeigte. Sie war nicht nur in Stanford die erste Ingenieurin, sondern auch in Leoben die erste Doktorin der Montanistischen Wissenschaften.

Durch Freunde fand sie eine erste Anstellung für neun Jahre bei der Crucible Steel Corporation, doch bald fand sie die Lebensstellung bei der Climax Molybdenum Company in New York, bei der sie bis zu ihrem Tode 1974 blieb. Unter den zahlreichen Publikationen ist "Archer/Briggs, Molybdenum-Steel, Iron and Alloys 1947“ [9] wohl am bekanntesten.

\section{Nachbemerkungen [NB1-2]}

1. [NB1] Jontes L. hat durch ihren Artikel [8] das Interesse des Autors an diesem Thema geweckt. Sie besitzt das Buch [4] mit der ausführlichen Biographie und auch kurze Beschreibungen ihrer wissenschaftlichen Arbeiten. Frau Hofrätin Dr. Jontes sei für die Möglichkeit der Verwendung dieser Literaturquelle herzlich gedankt. Dr. mont. Briggs wurde von ihr auch bei der Ausstellung zum 175- 


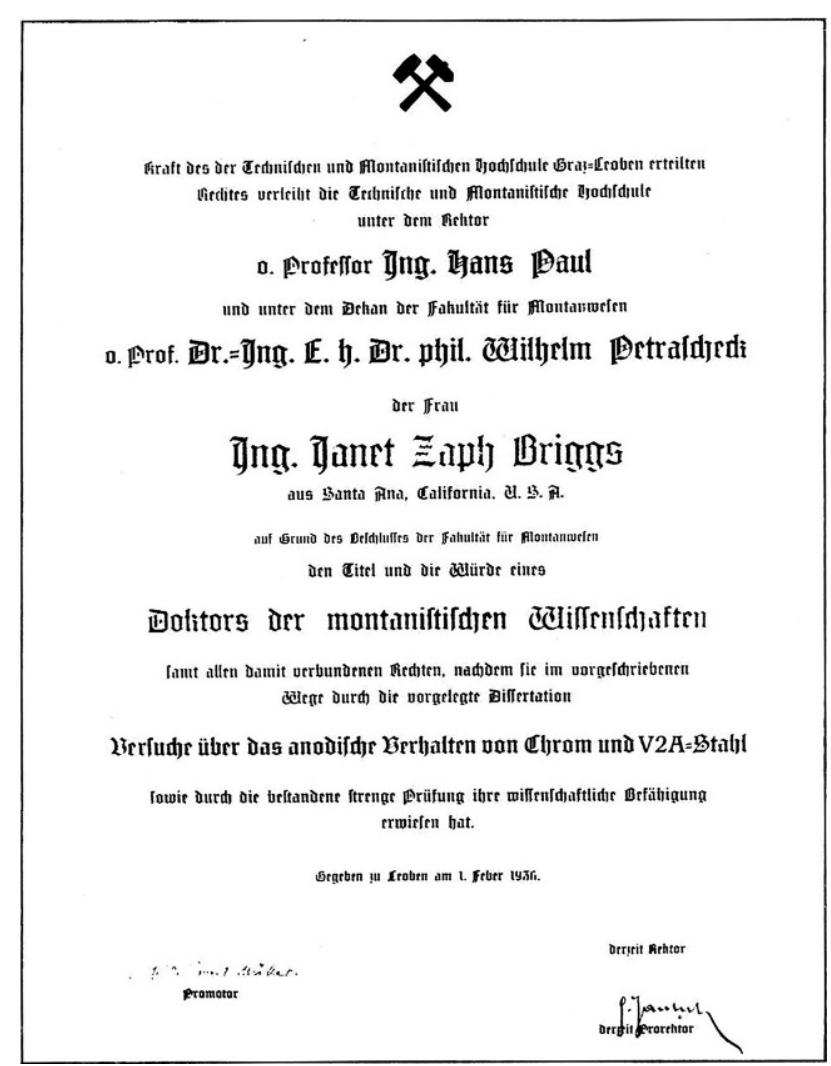

Abb. 5: Das Zeugnis über die Promotion in Leoben am 1. Februar 1936

Jahrjubiläum mit ihrem Doktordiplom in der Gangausstellung an der Montanuniversität kurz vorgestellt.

Da die direkte Beschaffung wegen Sperre der Arbeit in Stanford nicht möglich war, bat der Autor einen früheren Arbeitskollegen aus Menlo-Park, Kalifornien, Dr. Donald A. Shockey, im nahen Stanford Research Institute um Vermittlung, sodass diese Arbeit [2] nun als pdf-Datei und Kopie in Leoben beim Autor zur Einsichtnahme verfügbar ist. Das Originalzitat in Stanford ist: A short study of the making, working, and properties of ancient iron [1933], Briggs, J. Z. (Janet Zaph), 1912-1974. 1933. BOOK87, xviii leaves: ill., photos.; $28 \mathrm{~cm}$. Earth Sciences Library (Branner).

2. [NB2] Die Leobner Montanistische Hochschule (heute Montanuniversität) verlieh am 3. März 1933 dem Präsidenten der Vereinigten Staaten Ing. Herbert Clark Hoover (1874-1964) das Ehrendoktorat. Er musste sich durch den Gesandten Gilchrist Stockton vertreten lassen, da er am 4. März 1933 seinen Nachfolger als Präsidenten, Franklin Delano Roosevelt, zu dessen Angelobung begleiten sollte. Hoover, Sohn eines Schmiedes in lowa, hatte, wie später Janet Z. Briggs, an der Stanford Universität Bergbau (und Hüttenwesen) studiert und war als Berater und
Organisator von Bergwerken in allen Weltteilen unterwegs gewesen. Nach seiner Präsidentschaft engagierte er sich besonders auf caritativem Gebiet.

Sein Interesse an der Montangeschichte schlug sich darin nieder, dass er sich auf den langen Schiffsreisen vor seiner Präsidentschaft mit der Übersetzung des Buches Georg Agricolas, De re metallica libri XII [10] beschäftigte, wobei inm seine Frau Lou Henry, die Latein studiert hatte, bei der Übersetzung der Erstausgabe von 1555 behilflich war. Seine Kommentare darin sind von höchster Genauigkeit und übertreffen die Erstausgabe in modernem Deutsch von 1928 [11] in der Ausführlichkeit. Die originale Übersetzung von 1912 lag nur in wenigen Exemplaren vor, sie wurde aber u. a. 1950 in New York gedruckt herausgegeben [10].

Funding. Open access funding provided by Montanuniversität Leoben.

Open Access Dieser Artikel wird unter der Creative Commons Namensnennung 4.0 International Lizenz (http://creativecommons.org/licenses/ by/4.0/deed.de) veröffentlicht, welche die Nutzung, Vervielfältigung, Bearbeitung, Verbreitung und Wiedergabe in jeglichem Medium und Format erlaubt, sofern Sie den/die ursprünglichen Autor(en) und die Quelle ordnungsgemäß nennen, einen Linkzur Creative Commons Lizenz beifügen und angeben, ob Änderungen vorgenommen wurden.

\section{Literatur}

1. Sperl, G.: Hundert Jahre Schmelzversuche zur frühen Eisenerzeugung, Eisengewinnung und-Verarbeitung in der Frühzeit, LGH, Neue Folge, 2 (1981), S. 93-99

2. Briggs, J. Z.: A short Study of the Making, Working, and Properties of Ancient Iron, Diplomarbeit an der Stanford University California, 1933

3. Briggs, J. Z., Versuche über das anodische Verhalten von Chrom und V2A-Stahl, 2 Bände., Diss., Leoben, 1936

4. Barr, R. O.: The amazing J. Z. Briggs, Greenwich, Connecticut: Climax Molybdenum Company Division of AMAX Inc., 1974

5. Fettweis, G. B. L.: Vor 75 Jahren: Verleihung des Dr. mont. h.c. an den bedeutenden Bergingenieur und damaligen Präsidenten der Vereinigten Staaten von Amerika Herbert Clark Hoover, Berg- und Hüttenmännische Monatshefte, 153 (2008), Nr 4, S. 159-160

6. Sperl, G.: Zur Herstellung der Stahlsäule in Delhi (Indien), 4.Jahrhundert n. Chr., res montanarum, 50 (2012), S. 84-87 sowie Bergknappe, 2 (2013), S. 14-17

7. Briggs, J. Z., in: [2], S. 17 Indian Process for Wrought Iron: Lit. 71: Gowland, W.: The Metals in Antiquity, Journal of Royal Anthropological Institute, 42 (1912), S. 235-287

8. Jontes, L.: Von Leoben in die Welt. Bemerkenswerte Absolventen und Studierende an der Montanuniversität Leoben und ihrer Vorgänger S. 74-103, in: res montanarum, Fachzeitschrift des Montanhistorischen Vereins Österreich, Sonderband 3/2015, ISSN 1727-1797, hier: S. 89-90.

9. Archer, R. S.; Briggs, J. Z., Loeb, C. M., Jr.:Molybdenum-steels, irons, alloys, 394 Seiten, New York: Climax Molybdenum Comp., 1947

10. Hoover, H. C.; Hoover, L. H.: Georgius Agricola De Re Metallica; Druck der Übersetzung von 1912, New York: Dover Publ., 1950

11. Agricola, G.:1494-1555, Zwölf Bücher vom Berg- und Hüttenwesen, Berlin: VDI-Verlag, 1928 (und mehrere Nachdrucke davon) 